\begin{tabular}{|c|l|}
\hline Title & Distribution of cooperative unit size of amphi philic molecules in the phase coexistence region in Langmuir monol ay ers \\
\hline Author(s) & Hatta, E.; Nishimura, T. \\
\hline Citation & $\begin{array}{l}\text { Journal of Colloid and Interface Science, 391, 111-115 } \\
\text { https://doi.org/10.1016/.jcis.2012.09.074 }\end{array}$ \\
\hline Issue Date & 2013-02-01 \\
\hline Doc URL & http://hdl.handle.net/2115/51770 \\
\hline Type & article(author version) \\
\hline File Information & JCIS391_111-115.pdf \\
\hline
\end{tabular}

Instructions for use 


\title{
Distribution of cooperative unit size of amphiphilic molecules in the phase coexistence region in Langmuir monolayers
}

\author{
E. Hatta ${ }^{*}$ and T. Nishimura
}

Nanoelectronics Laboratory, Graduate School of Information Science and Technology, Hokkaido University, Sapporo, 060-0814, Japan

The dependence of the size of cooperative unit (C.U.) of the amphiphilic molecules on the surface pressure $(\pi)$ in the liquid expanded (LE) - liquid condensed (LC) phase coexistence region of Langmuir monolayers has been formulated and calculated using the measured isotherm data. The C.U. size changes largely depending on the surface pressure in the coexistence region and these submicroscopic molecular aggregates are not static objects but dynamic ones characterized by large fluctuations in size. It has been found that the C.U. size distribution can be a natural consequence from the significant change of monolayer compressibility, which reflects the large molecular area density fluctuations, in the coexistence region.

Keywords: Langmuir monolayers, Phase transition, Isotherm, Compressibility, Cooperative unit

\footnotetext{
*Email: hatta@nano.ist.hokudai.ac.jp
} 


\section{Introduction}

The nonhorizontal nature of the isotherms in the phase coexisting region of the liquid expanded (LE) - liquid condensed (LC) phase transition in Langmuir monolayers has been the subject of a great deal of experimental and theoretical work for decades $[1,2]$. Pallas and Pethica found a horizontal plateau and concluded that the transition is first order [3]. This result is consistent with the results of microscopy experiments in which an isothermal coexistence of LE and LC domains was observed [4]. The nonhorizontal plateau found by most researchers under normal compression conditions is, however, different from that of the conventional first order transition [5]. This nonhorizontal isotherm slope has been associated with some possible sources. Some authors have suggested that the impurities may be the cause of the absence of horizontal curves [3, 6]. Arriaga et al. discussed the relation between the nonhorizontal plateau and the finite rigidity of a microheterogeneous medium composed of two coexisting phases [7]. The possibility of polar-headgroup interactions [8] and interphase strain effects [9] were also invoked to explain the experimentally observed nonhorizontal isotherms. Besides the above experimental viewpoints, present theoretical treatments of nonhorizontal isotherms are based on discontinuous transitions with the formation of two-dimensional molecular aggregates such as surface micelles [10] or small molecular clusters (or 
submicroscopic domains) [11]. One should here remember that the sharpness of a phase transition depends on the number of molecules forced to cooperate in the transition [12]. This number of molecules is called the cooperative unit (C.U.) of the transition [13]. In an exact thermodynamic treatment a first-order phase transition is defined as a transition for which there is a discontinuity in thermodynamic function. Such sharp transitions only occur for an infinite C.U.. For small, finite systems, the C.U. size is finite, and the range of coexistence temperatures (or pressures) is also finite. Since lipid bilayer vesicles and monolayers always have a finite number of molecules, transition widths become never infinitely small. The previous analysis of the C.U. size has been normally based on the assumption that it is kept constant during the transition $[10,11,14]$; however, the validity of this assumption remains unclear $[15,16,17]$. A more sophisticated analysis of such clusters must thus be given. In this paper a simple procedure has been developed in order to calculate the C.U. size distribution during the LE-LC transition using the measured isotherm data. The C.U. size has been found not to be kept constant but to be a function of surface pressure in the coexistence region. The present analysis demonstrates that large fluctuations in molecular area density, which is manifested by the distribution of isothermal lateral compressibility, lead to the strong pressure dependence of the C.U. size in the coexistence region. 


\section{Theory}

Within the pressure interval of phase transition, equilibrium is established if the compression is made slowly enough. One may assume that monolayers consist of only LE and LC domains during the transition. Then the reversible process of LE-LC transition can be considered as a process of transformation of such domains to each other with speeds proportional to density of domains. In other words the phase equilibrium can be considered as a two-state, "all-or-none" transition [18, 19]:

$$
[L E] \stackrel{K}{\leftrightarrow}[L C],
$$

with the two-state, isothermal equilibrium constant,

$$
K=\frac{[L C]}{[L E]},
$$

where $[L E]$ and $[L C]$ are the densities of molecules in LE and LC phases, respectively.

We note that the "all-or-none" transition is a microscopic analogue of the first-order phase transition in macroscopic systems. Unlike true phase transitions, the "all-or-none" transitions have a non-zero width of the pressure in the transition, since this transition embraces a system with a small, finite number of molecules. In the two-state "all-or-none" transition, the fraction of the molecules in the LC phase, i.e., the order parameter, $\xi$ can be defined thermodynamically with a two-state isothermal equilibrium constant $K$. 


$$
\xi \equiv \frac{[L C]}{[L E]+[L C]}=\frac{K}{1+K}, \quad\left(K=\frac{\xi}{1-\xi}\right) .
$$

As the surface pressure increases, there will be a shift in the fraction of molecules in the LC phase, dictated by the pressure dependence of the equilibrium constant. A transition with a high cooperativity means that $\xi$ changes from very small to very large over a very small surface pressure range, and one can expect a very strong surface pressure dependence to the equilibrium constant. In lipid bilayers, for instance, the temperature range of the gel to liquid crystalline transition is narrow, but finite, suggesting a significant degree of cooperativity. Let us assume that molecule do not undergo the transition independently of each other but simultaneously in clusters of $n$ molecules. Then we have to consider the cluster of these $n$ molecules as the C.U.. The change of free energy per C.U. size at transition $n \Delta G$ ( $\Delta G$ : the difference of Gibbs free energy per molecule) can be related to the equilibrium constant:

$$
\ln K(\pi, T)=-\frac{n \Delta G(\pi, T)}{k T}
$$

From fundamental equation in thermodynamics,

$$
d G=-S d T+a d \pi
$$

At a constant temperature, from eq. (4) and eq. (5), the pressure dependence of $K(\pi)$ is as follows:

$$
K(\pi)=\exp \left(-\frac{n \Delta a\left(\pi-\pi_{m}\right)}{k T}\right)
$$


where $\pi_{m}$ is the surface pressure at the midpoint of the transition.

Next, let us derive the relation between the isothermal area compressibility, $\kappa_{T}^{A}$ and the surface pressure dependent variable C.U. size, $n(\pi)$. The isothermal area compressibility is defined as follows [20]:

$$
\kappa_{T}^{A}(\pi)=-\frac{1}{a}\left(\frac{\partial a}{\partial \pi}\right)_{T} .
$$

We note the relation below:

$$
\left(\frac{\partial \xi}{\partial a}\right)_{T}=\left(\frac{\partial \xi}{\partial \pi}\right)_{T}\left(\frac{\partial \pi}{\partial a}\right)_{T}
$$

If a monolayer occupies a molecular area $a$ located within the LE - LC coexistence region, then the order parameter $\xi$, e.g., the molecular number fraction of the system that is in the LC phase can be given by the application of the "lever rule".

$$
\xi \cong \frac{a-a_{L E}}{a_{L C}-a_{L E}}=\frac{1}{\Delta a} \cdot\left(a-a_{L E}\right)
$$

The left-hand side of eq. (8) can thus be approximated utilizing the above equation (9).

Finally, from eqs. (6) - (9), we can obtain the following relation.

$$
\begin{aligned}
\kappa_{T}^{A}(\pi) & =-\frac{1}{a}\left(\frac{\partial a}{\partial \pi}\right)_{T} \\
& =-\frac{\Delta a}{a}\left(\frac{\partial \xi}{\partial \pi}\right)_{T} \\
& =-\frac{\Delta a}{a}\left(\frac{\partial}{\partial \pi}\left(\frac{K}{1+K}\right)\right)_{T} \\
& =\frac{n(\Delta a)^{2}}{a k T} \cdot \frac{\exp \left(-\frac{n \Delta a\left(\pi-\pi_{m}\right)}{k T}\right)}{\left(1+\exp \left(-\frac{n \Delta a\left(\pi-\pi_{m}\right)}{k T}\right)\right)^{2}}
\end{aligned}
$$


Using the above equation (10) into which the measured isotherm data can be incorporated, one can calculate the C.U. size $n(\pi)$ distribution during the LE-LC phase transition at a constant $T$ numerically.

\section{Experimental}

Pentadecanoic acid (PDA) was purchased from Sigma and claimed to be $99+\%$ pure. It was used without further purification. A high surfactant concentration $(5 \mathrm{mMol} / \mathrm{l})$ was used to minimize the possibility of solvent (n-hexane, Merck) contamination and therefore a small volume of solution $(5 \mu \mathrm{l})$ was spread onto pure water (Millipore Milli-Q at $18 \mathrm{M} \Omega \mathrm{cm}, \mathrm{pH} 5.5$ ) contained in a custom-built Teflon trough. After n-hexane was allowed to evaporate for $20 \mathrm{~min}$, the PDA monolayers were compressed at a rate of $1 \AA$ molecule $\mathrm{emin}^{-1}$. Thermocouples immersed in the subphase were used to measure the subphase temperature and the surface pressure was measured with a filter paper Wilhelmy plate in conjunction with an $R \& K$ transducer. The surface pressure sensibility was $0.1 \mathrm{mN} / \mathrm{m}$. The monolayer surface pressure $(\pi)$ and the molecular area $(A)$ were automatically stored on disk. The isothermal area compressibility $\kappa_{T}{ }^{A}$ was calculated from the digitally stored data. The copper bottom was coated with a $0.3 \mathrm{~mm}$ thin Teflon foil. The subphase temperature was controlled by two water cooled Peltier elements mounted directly on the back side of the copper plate. 


\section{Results and discussion}

4.1. Evaluation of the cooperative unit size in the LE-LC phase coexistence region of PDA monolayers

The $\pi-\mathrm{A}$ isotherms of pentadecanoic acid (PDA) monolayers at the air-water interface are shown in Fig. 1. The LE-LC phase transition, characterized by the abrupt decrease in molecular area, can be seen on the isotherms. As the subphase temperature increases, the onset area of the transition became smaller and the molecular area range where the two phases coexist became narrower. These characteristics are in agreement with the results previously reported $[1,3]$. The nonhorizontal plateau in the two phase

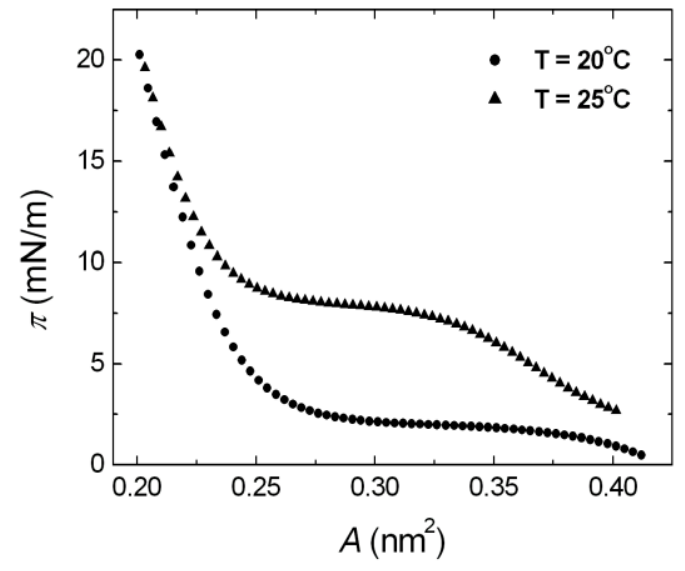

Figure 1 Surface pressure $(\pi)-$ molecular area $(A)$ isotherms of pentadecanoic acid monolayers at different temperatures.

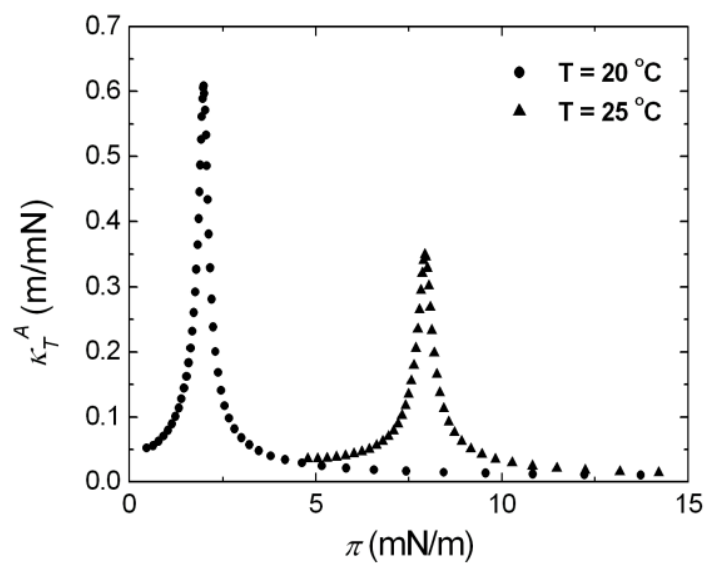

Figure 2 Isothermal lateral compressibility $\left(\kappa_{T}^{A}\right)$ - surface pressure $(\pi)$ characteristics of pentadecanoic acid monolayers at different temperatures. 
coexistence region was observed in the both isotherms. The $\kappa_{T}{ }^{A}-\pi$ characteristics were calculated from the isotherms of Figure 1, being shown in Fig. 2. They showed pronounced peaks that shifted toward higher pressures with increasing temperatures. The distribution of C.U. size of molecules $(n)$ was calculated as a function of surface pressure, $\pi$, using the measured isotherm data $\left(a, \pi, \kappa_{T}{ }^{A}\right)$ and eq. (10) (Fig. 3). The transition midpoint pressure $\pi_{m}(T)$ was determined as the peak point on the compressibility curves of Fig. 2. The C.U. size curves showed peaks corresponding to $n=195\left(\approx 65 \mathrm{~nm}^{2}\right.$ in cluster area) and $174\left(\approx 51 \mathrm{~nm}^{2}\right)$ with finite half-widths of transition surface pressure $\left(\Delta \pi_{\text {half }}=0.49\right.$ and $\left.0.66 \mathrm{mN} / \mathrm{m}\right)$, at 20 and $25{ }^{\circ} \mathrm{C}$, respectively. Here the cluster area, $a_{\text {cluster }}$, was calculated using the relation,

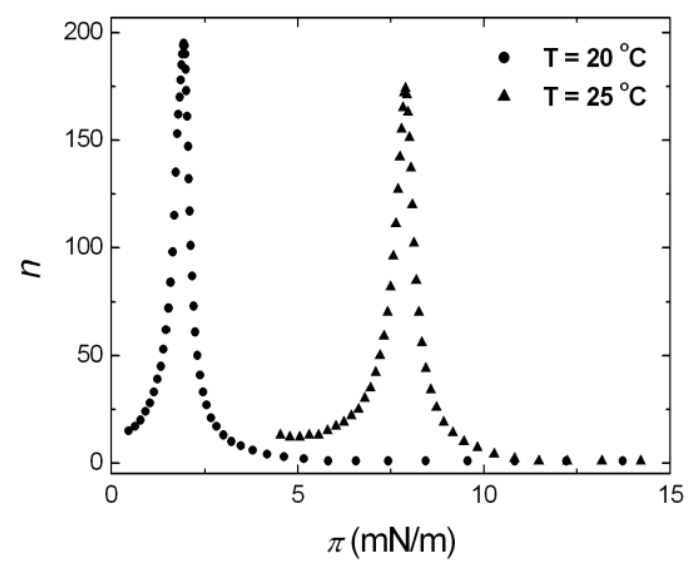

Figure 3 Dependence of the calculated C.U. size $(n)$ on the surface pressure $(\pi)$ for pentadecanoic acid monolayers at different temperatures. 
$a_{\text {cluster }} \approx n \cdot a_{n}$, where $n$ is the C.U. size and $a_{n}$ is the molecular area at the $n$. From the data of Fig. 1 and Fig. 3 one can obtain $a_{n}=0.33$ and $0.29 \mathrm{~nm}^{2}$ at 20 and $25{ }^{\circ} \mathrm{C}$, respectively.

4.2. The connection among isothermal compressibility, lateral density fluctuation,

\section{and cooperative unit}

Since monolayers are quasi-two-dimensional systems, large fluctuations in long range order are expected. In a standard statistical thermodynamics treatment [21], it can be shown that the isothermal compressibility is directly related to fluctuations in density in the system by the following equation:

$$
\frac{k T \kappa_{T}^{A}}{a}=\frac{\overline{\left(\rho_{A}-\overline{\rho_{A}}\right)^{2}}}{{\overline{\rho_{A}}}^{2}},
$$

where the right-hand side of the above equation is the mean square relative deviation in area density from the mean area density. One can see from this relation that large lateral density fluctuations in the system lead to the large isothermal compressibility. The present analysis thus leads to some insight on the molecular level by revealing the distributions of C.U. size in terms of lateral compressibility, i.e., the C.U. size is a measure of coherence length of lateral density fluctuations (, or dynamic lateral heterogeneity). The advantage of this simple procedure that can calculate the C.U. size distribution in the LE-LC transition is that the physical quantities $\left(\kappa_{T}, a, \Delta a, \pi, \pi_{m}\right)$ 
incorporated to calculate the variable unit size are all experimentally accessible and no adjustable parameters are required. Another advantage of this approach is that the dynamic character of cooperative clusters during the transition is manifested by the existence of large lateral density fluctuations in the monolayer through the lateral compressibility. In the phase transition of the system with a finite number of molecules, the limit of cooperativity is such that all the molecules of the system go between the two states together at a single pressure. The LE-LC transition has characteristics different from those of the conventional first-order phase transition, since those occur from the long-range repulsive interactions among amphiphilic molecules, which induces the formation of LC islands dispersed in a continuous phase of LE molecules. Generally due to the heterogeneity and the existence of discrete domains in the monolayers, the size of the C.U. tends to be less than the number of molecules constituting monolayers. A true first-order, discontinuous phase transition (, therefore $\kappa_{T}^{A} \rightarrow \infty$ ) occurs only for an infinite C.U.. Thus in the system of larger C.U.'s, the range of pressures over which LE and LC phases coexist, is narrow, and so the smaller the pressure range over which the system has a compressibility larger than in the single (LE or LC) phase. The larger the C.U., however, the larger will be the increase in compressibility over that in the single phase. These are consistent with our results (Fig. 2 and Fig. 3). 


\subsection{Comparison with other studies}

In [18], the slope of the isotherm was assumed to be a straight line in the coexisting region, and the order of 80-150 molecules was given as the constant C.U. size at each temperature in the transition. We must note that the slope of isotherm is generally not a straight line but a curved one in the transition region, as observed by most researchers. This suggests that the C.U. size must depend on the molecular area (, and therefore the surface pressure) during the transition [15, 16, 17]. Calculations of the pressure dependent, C.U. size $n(\pi)$ must be thus important. The C.U. size serves as a simple comparative characteristic parameter of the phase transitions evaluated by different methods under different conditions. The values of unit size $n$, have been calculated mainly using the constant C.U. approach from DSC data in the phase transitions of lipid bilayer systems [22,23,24]. For dipalmitoylphosphatidylcholine (DPPC) they are of the order of several hundreds for multilamellar vesicles and several tens for unilamellar vesicles. There have been, on the other hand, very few reports on the size dependence of C.U. on some intensive variable (e.g., temperature, pressure) during the phase transition $[25,26]$. The size of C.U.'s (the order of $10 \mathrm{~nm}^{2}$ ) estimated in our study is much smaller than that of domains (typically, the order of $10-100 \mu \mathrm{m}^{2}$ ) normally observed by Brewster angle microscopy (BAM) and fluorescence microscopy (FM). It lies in a range 
of cluster sizes beyond the resolution of standard light microscopy techniques. Fischer and Sackmann observed the coexistence of fluid and solid domains with the size of the order of a few hundred lipid molecules from transmission electron microscopy and electron diffraction of monolayers transferred onto solid substrates in the two-phase coexistence region [27]. Grazing incidence diffraction of neutron study provided cluster sizes of less than $10 \mathrm{~nm}$ in the coexistence phase of binary phospholipid mixture [28]. This result is comparable to our results. There is a rapidly growing number of direct observations of gel and fluid clusters in binary lipid mixtures by atomic force microscopy (AFM) [29,30]. There are, however, vast differences between the observed cluster sizes and shapes depending on the type of the measurement, the construction, and thermal history of the sample. The AFM measurements on one layer of supported bilayer revealed gel cluster sizes from $10 \mathrm{~nm}$ to several micrometers. It should be noted in AFM measurements on the phase transition of supported lipid bilayers that the resultant stress introduced into the gel phase during the transition strongly changes the degree of cooperativity [31]. Recently the phase transition from the $L_{\alpha}$-phase to the $P_{\beta^{\prime}}$-phase of dimyristoylphosphatidylcholine (DMPC) was studied at high temperature resolution $(\Delta T \leq 0.1 \mathrm{~K})$ by $\mathrm{AFM}[32]$. The representative image indicated that the molecules of a C.U. are organized in small lipid domains. The unit size $n$ could be 
determined from pairs of consecutive images in the nm-scale of the transition by correlation analysis. The mean value of $n$ was obtained of 195 molecules. The value is of the order of magnitude as derived by us. A possible dependence of the C.U. size on temperature was pointed out also in that study.

\section{Conclusions and outlook}

In this paper we have formulated and calculated the C.U. size distribution during the LE-LC phase transition in Langmuir monolayers using a simple two-state model. The midpoint surface pressure, transition area width, and area compressibility were incorporated into the equation from the measured isotherms. From our combined experimental and theoretical approach, it has been found that the calculated C.U. size is not kept constant but changes largely depending on the surface pressure in the coexistence region. The distribution of the C.U. size reaches a maximum at $\pi_{\mathrm{m}}$ in the transition region. It has been clarified that formation of C.U. clusters is closely related to lateral density fluctuations and isothermal lateral compressibility. The present study gives an insight into a significant role of fluctuations accompanying the phase transition in monolayers. Since the C.U. size is a measure of the coherence length of two-dimensional molecular density fluctuations through the isothermal compressibility, it is clear that the LE-LC transition is accompanied by the lateral density fluctuations 
that change largely during the transition. Experimental observations have been presented on the phase transition dependence of biological functions of the lipid membrane. The passive permeability of lipid membranes for ions and molecules displays a maximum close to the chain melting transition of the membranes [33]. The enzyme activity of phospholipase $A_{2}$ is at a maximum in the phase coexistence region near the gel-fluid phase transition temperature of the vesicular bilayers [34]. The above enhanced physical properties were attributed to the degree of lateral heterogeneity of the lipid bilayer in the transition region. The degree of lateral heterogeneity in the lipid bilayer is closely related to the lateral density fluctuations that are in turn related to the C. U. size. Our results thus corroborate their studies since they give a certain quantitative measure of lateral heterogeneity in the transition region. Biological membranes consist predominantly of lipid bilayers with proteins and sterols imbedded in this structure. The lateral organization of lipids in biological and model membranes has been studied intensively in recent years, since it has been shown to be relevant for various functions, including protein activities, vesicle budding, and membrane trafficking [35]. The importance of lipid organization in such biological processes emphasizes the need to understand the factors that regulate domain formation and stability, and the relevant size of the lipid structures formed [36]. Studies of the physical 
properties near the phase transition are very important in understanding structure-function relationships in biological membranes, since phase transitions play important roles in many biological functions, as is clear from above examples $[33,34]$. From the above viewpoints, as the further extension of the present study, it would be of particular interest to investigate how the cholesterol and the different classes of proteins incorporated into the lipid monolayer matrix affect the cooperative nature, e.g. lipid organization and dynamic lateral heterogeneity of the monolayer in the transition region using the present approach $[37,38]$. The effect of size fluctuations in collective clusters is lacking of the mean field description of the first-order phase transition and therefore the large lateral density fluctuations in the transition must play a significant role in the occurrence of nonhorizontal plateau in monolayer isotherms. The approach presented in this study may serve as a useful method for the study of thermodynamic details of the phase transition in monolayers. 


\section{Figure Captions}

Figure 1 Surface pressure $(\pi)$ - molecular area $(A)$ isotherms of pentadecanoic acid monolayers at different temperatures.

Figure 2 Isothermal lateral compressibility $\left(\kappa_{T}^{A}\right)$ - surface pressure $(\pi)$ characteristics of pentadecanoic acid monolayers at different temperatures.

Figure 3 Dependence of the calculated C.U. size $(n)$ on the surface pressure $(\pi)$ for pentadecanoic acid monolayers at different temperatures. 


\section{References}

[1] W. D. Harkins, The Physical Chemistry of Surface Films, Reinhold, New York, 1952.

[2] V. M. Kaganer, H. Möhwald, P. Dutta, Rev. Mod. Phys. 71 (1999) 779.

[3] N. R. Pallas, B. A. Pethica, Langmuir 1 (1985) 509.

[4] M. Lösche, E. Sackmann, H. Möhwald, Ber. Bunsenges. Phys. Chem. 87 (1983) 848.

[5] T. L. Hill, An Introduction to Statistical Thermodynamics, Addison-Wesley, Boston, 1960.

[6] Y. H. Hifeda, G. W. Rayfield, Langmuir 8 (1985) 197.

[7] L. R. Arriaga, I. López-Montero, J. Ignés-Mullol, F. Montroy, J. Phys. Chem. B 114 (2010) 4509.

[8] A. Fischer A, M. Lösche, E. Sackmann, J. Phys.Lett. 45 (1984) L785.

[9] F. Nagle, Faraday. Discuss. Chem. Soc. 81 (1986) 151.

[10] J. Israelachvili, Langmuir 10 (1994) 3774.

[11] E. Ruckenstein and B. Li, Langmuir 11 (1995) 3510.

[12] T. L. Hill, Thermodynamics of small systems, Part 1, W. A. Benjamin, New York, 1963.

[13] D. T. Haynie, Biological Thermodynamics, 2nd, Cambridge University Press, New 
York, 2008.

[14] H. Heerklotz, A. Tsamaloukas, K. Kita-Tokarczyk, P. Strunz, T. Gutberlet, J. Am. Chem. Soc. 126 (2004) 16544.

[15] T. Heimburg, Thermal Biophysics of Membranes, WILEY, Weinheim, 2007.

[16] C. Johann, P. Garidel, L. Mennicke, A. Blume, Biophys. J. 71 (1996) 3215.

[17] L. K. Nielsen, T. Bjørnholm, O. G. Mouritsen, Nature, 404 (2000) 352.

[18] O. Albrecht, H. Gruler, E. Sackmann, J. Phys. (Paris) 39 (1978) 301.

[19] B. Gruenewald, A. Blume, F. Watanabe, Biochim. Biophys. Acta 41 (1980) 597.

[20] G. L. Gaines, Insoluble Monolayers at Liquid-Gas Interfaces, Interscience: New York, 1966.

[21] L. D. Landau, E. M. Lifshitz, Statistical Physics, 2nd, Pergamon, Oxford, 1969.

[22] S. Mabrey, J. M. Sturtevant, Proc. Natl. Acad. Sci. USA 73 (1976) 3862.

[23] N. Albon, J. M. Sturtevant, Proc. Natl. Acad. Sci. USA 75 (1978) 2258.

[24] H. -J. Hinz, J. M. Sturtevant, J. Biol. Chem. 247 (1972) 6071.

[25] I. P. Sugár, J. Phys. Chem. 91 (1987) 95.

[26] R. L. Biltonen, J. Chem. Thermodynamics 22 (1990) 1.

[27] A. Fischer, E. Sackmann, J. Phys. (Paris) 45 (1984) 517.

[28] C. Gliss, H. Clausen-Schaumann, R. Günther, S. Odenbach, O. Randl, Biophys. J. 74 (1998) 2443.

[29] B. Seantier, M. C. Giocondi, C. Le Grimellec, P. E. Milhiet, Curr Opin Colloid Interface Sci. 13 (2008) 326.

[30] E. I. Goksu, J. M. Vanegas, C. D. Blanchette, W. -C. Lin, M. L. Longo, Biochim. 
Biophys. Acta 1788 (2009) 254.

[31] A. F. Xie, R. Yamada, A. A. Gewirth, S. Granick, Phys. Rev. Lett. 89 (2002) 246103.

[32] O. Enders, A. Ngezahayo, M. Wiechmann, F. Leisten, H. -A. Kolb, Biophys. J. 87 (2004) 2522.

[33] A. Blicher, K. Wodzinska, M. Fidorra, M. Winterhalter, T. Heimburg, Biophys. J. 96 (2009) 4581.

[34] T. Hønger, K. Jørgensen, R. L. Biltonen, O. G. Mouritsen, Biochem. 35 (1996) 9003.

[35] K. Simons, W. L. Vaz, Annu. Rev. Biophys. Biomol. Struct. 33 (2004) 269.

[36] K. Jacobson, O. G. Mouritsen, R. G. W. Anderson, Nature Cell Biol. 9 (2007) 7.

[37] J. R. Silvius, Biochim. Biophys. Acta 1610 (2003) 174.

[38] B. Maggio, G. A. Borioli, M. Del Boca, L. De Tullio, M. L. Fanani, R. G. Oliveira, C. M. Rosetti, N. Wilke, Cell Biochem. Biophys. 50 (2008) 79. 